\title{
Création de modèles animaux de maladies humaines héréditaires
}

La disposition de modèles animaux de maladies humaines est essentielle à nombre d'études physiologiques et thérapeutiques de ces maladies. Ces modèles peuvent être souvent créés lorsqu'il s'agit d'affections acquises (maladie infectieuse, diabète, encéphalite expérimentale, etc.). En revanche, jusqu'à présent les seuls modèles utilisables de maladies héréditaires étaient ceux que produisait la nature... éventuellement " aidée " par un traitement mutagène des animaux (par exemple le déficit en ornithine transcarbamylase de la souris [1]. Ces modèles étaient malheureusement peu nombreux et souvent peu représentatifs des maladies humaines. L'introduction de la méthodologie des animaux transgéniques a permis d'envisager non seulement la "thérapie génique " d'affections dues à l'absence ou à l'insuffisance d'expression d'un gène $(\mathrm{m} / \mathrm{s}$ $n^{\circ} 10$, vol. 2, p. 579), mais aussi la création de modèles de maladies héréditaires dues à l'expression d'un gène qualitativement modifié ; nous avons ainsi rapporté qu'il était désormais possible de développer un modèle murin de drépanocytose en injectant le gène humain $\beta^{s}$ dans des œufs de souris $B$ thalassémiques $\left(\mathrm{m} / \mathrm{s} n^{\circ} 1\right.$, vol. 3, p. 49).

Les signes cliniques d'une autre maladie, le déficit en $\alpha 1$-antitrypsine, sont dus d'une part à l'absence de l'activité enzymatique circulante, et d'autre part à l'accumulation dans le foie de la protéine mutée PiZ dont le transport intracellulaire est modifié $(\mathrm{m} / \mathrm{s}$ $n^{\circ} 3$, vol. $\left.3, p .181\right)$. Des souris transgéniques pour l' $\alpha 1$-antitrypsine humaine ont été récemment obtenues par injection du gène normal [2]. Le gène muté PiZ ayant été cloné, il devrait être possible d'obtenir de la même exprimant très activement la protéine mutée humaine et d'étudier alors son accumulation dans le foie et les dégâts tissulaires ainsi provoqués.

D'une certaine manière, on peut considérer que les animaux transgéniques pour un oncogène activé sont aussi des modèles de cette maladie génétique acquise qu'est le cancer.

La création de modèles vrais de maladies en rapport avec un déficit en une activité biologique, tel un déficit enzymatique, pose en revanche des problèmes beaucoup plus complexes: un transgène peut compenser une activité absente... et non directement supprimer une activité présente. Une solution à laquelle de nombreuses équipes avaient pensé était de produire des "phénocopies" d'une mutation en introduisant un transgène transcrit en un ARN antisens capable de bloquer l'expression de l'ARN "sens" normal (m/s $n^{\circ} 5$, vol. 3, p. 299). Nous avons vu dans le dernier numéro de $\mathrm{m} / \mathrm{s}$ que cette stratégie, théoriquement attrayante, était en fait rarement couronnée de succès. Dans le numéro de Nature daté du 19-3-1987, une toute nouvelle stratégie apparaît, combinant les possibilités de sélection de mutants dans des cellules en culture et l'intérêt physiologique des modèles animaux. Des cellules souches embryonnaires totipotentes sont cultivées sur un milieu sélectif permettant de sélectionner des clones déficients en une enzyme de la voie d'épargne des purines, l'HGPR T (hypoxanthine guanylphosphoribosyl-transférase) : en l'absence de cette enzyme les purines libres ne sont pas utilisées, et par conséquent des analogues toxiques des purines tuent les cellules normales et non les cellules déficientes qui ne les incorporent pas dans leur ADN. Des cellules
HGPRT(-) ont ainsi été obtenues soit par mutation spontanée [3], soit par culture sur une couche de cellules produisant des rétrovirus, certains intégrant leur $A D N$ proviral au milieu du gène HGPRT [4]. La particularité des cellules souches embryonnaires totipotentes est de pouvoir se différencier en tous les types cellulaires et, intégrées dans un embryon précoce, de participer normalement à l'embryogenèse, donnant des animaux chimères dont certaines cellules somatiques et germinales sont dérivées de l'embryon précoce, d'autres des cellules totipotentes. Une à deux dizaines de cellules HGPRT $(-)$ ont ainsi été injectées dans les blastocystes de souris normales. Après réimplantation des embryons, une moyenne de $25 \%$ des souriceaux avaient une constitution chimérique bien mise en évidence par l'existence d'un pelage bicolore correspondant aux pelages des souches de souris donneuses de blastocystes et de cellules souches embryonnaires. Des croisements ultérieurs permirent d'aboutir à des femelles hétérozygotes et à des mâles hémizygotes pour la mutation HGPRT (-) (le gène HGPRT est porté par le chromosome $\mathrm{X}$, les femelles $\mathrm{X}$ normal/X muté étant hétérozygotes et les mâles $\mathrm{X}$ muté/Y étant hémizygotes et déficients).

Le déficit en HGPRT donne chez l'homme une maladie récessive liée au sexe appelée maladie de Lesch-Nyhan, caractérisée par une hyperuricémie sévère, une arriération mentale et une tendance singulière à l'automutilation. Jusqu'à présent, les souris mâles hémizygotes pour la mutation n'ont pas présenté de troubles comportementaux, mais ceux-ci n'apparaissent que secondairement dans la maladie humaine. En toute éventualité, ces résultats 
marquent manifestement une date importante dans les études de génétique pathologique ; il est en effet très souvent possible, grâce à la mise au point de systèmes sélectifs particuliers, de sélectionner des phénotypes cellulaires rares, conséquences de mutations spontanées ou provoquées qui peuvent n'intéresser qu'une cellule sur plusieurs millions. La possibilité maintenant démontrée de produire des animaux à partir de ces cellules mutées augmente dans des proportions immenses le champ de la génétique des mammifères [5].

A.K.

1. Cavard C, Grimbert G, Chasse JF, et al Correction d'un déficit enzymatique murin par transfert de gène. médecine/sciences $1987 ; 3$ : 38-40.

2. Sifers RN, Carlson JA, M Clift S, et al. Tissue-specific expression of the human $\alpha 1$-antitrypsin gene in transgenic mice. Nucl Acid Res 1987 ; 15 : 1459-74.

3. Hooper M, Hardy K, Handyside A, Hunter S, Monk M. HGPRT-deficient (LeschNyhan) mouse embryos derived from germline colonization by cultured cells. Nature 1987 ; $326: 292-5$.

4. Kuehn M, Bradley A, Robertson EJ Evans MJ. A potential animal model for Lesch Nyhan syndrome through introduction of HGPRT mutations into mice. Nature 1987 ; 326 : 295-8.

5. Hogan B. Engineering mutant mice. Nature $1987 ; 326: 240-1$.

\section{$\square \square$ BRÈVES}

Le réarrangement préalable des gènes d'immunoglobuline n'est pas indispensable à l'accumulation des mutations somatiques. De telles mutations s'accumulent en effet au niveau d'un transgène kappa non réarrangé ; elles n'intéressent cependant que les segments codant pour les régions variables, et non ceux codant pour les régions constantes. Ces résultats suggèrent la présence, au niveau des gènes d'immunoglobuline, d'un "mutateur" spécifique de certaines séquences.

[O'Brien PL, et al. Nature 1987 326 : 405-9.]

$\mathrm{m} / \mathrm{s} n^{\circ} 6$ vol. 3 , juin 87

\section{Le locus de la fibrose kystique identifié ?}

Le gène de la mucoviscidose (fibrose kystique du pancréas, CF), a été localisé en 1985 par plusieurs groupes sur le chromosome 7 en 7 q22-31. Les marqueurs les plus proches connus jusqu'à présent (J3.11 et l'oncogène met) encadrent probablement le locus $C F$. Le groupe londonien de Williamson a transféré de l'ADN contenant le gène met activé et vraisemblablement le locus CF dans des fibroblastes de souris par la technique de "transfert de chromosome". Relatant ces résultats en janvier dernier (m/s $\mathrm{n}^{\circ} 1$, vol. 3, p. 47), nous laissions espérer des progrès qui ont été encore plus rapides que prévu. La même équipe [1] a en effet réussi à préparer une lignée de cellules CIId, dont la contribution humaine est limitée à $10^{6}$ paires de bases, provenant du chromosome 7 et contenant met. Pour éviter une fastidieuse " marche sur le chromosome" à partir de met, les auteurs ont mis en auvre une technique basée sur une hypothèse de Bird [2]. De nombreux gènes codant pour des protéines sont précédés par une région d'ADN riche en dinucléotides CpG non méthylés, sensibles à des enzymes de restriction spécifiques dont le modèle est HpaII, d'où leur nom d'ilôts HTF (HpaII tiny fragments). On peut ainsi obtenir électivement des segments d'ADN contenant des gènes, que l'on insère dans des cosmides. Au terme d'une procédure complexe, deux clones $X V-2 a$ et CS7 ont été isolés ; ils répondaient aux critères des ilôts HTF et pouvaient être localisés entre J3.11 et met. Présentant un polymorphisme de restriction, ils ont permis une étude de liaison génétique dans des familles porteuses du gène $C F$. Le taux de recombinaison très faible, surtout pour le marqueur CS7, conduit à penser que la distance de CF à chacun des deux marqueurs ne dépasse pas 10 kilobases.

Deux arguments sont en faveur de l'existence d'un locus fonctionnel dans la région d'ADN étudiée. Les deux clones isolés s'hybrident à l'ADN de nombreux mammiferes et du poulet, mon- trant que ces séquences sont bien conservées. Surtout, un cosmide contenant ces clones s'hybride en Northern blot à des messagers abondants dans le poumon, le rein et l'intestin, distribution tissulaire qu'on pourrait attendre d'un gène CF. Il reste à en pratiquer l'identification définitive, et à en analyser les anomalies dans la mucoviscidose. Notons que, parmi ces anomalies, ne semblent pas figurer de délétions, contrairement à ce que l'on a trouvé dans la myopathie de Duchenne.

J.-C.D.

1. Estivill X, Farrall M, Scambler PJ, et al. A candidate for the cystic fibrosis locus isolated by selection for methylation-free islands. Nature $1987 ; 326: 840-5$.

2. Bird AP.CpG rich islands and the function of DNA methylation. Nature $1986 ; 321$ 209-13.

\section{$\square \square \square$ BRÈVES $\square \square \square$}

La protéine kinase $\mathrm{C}$ est inhibée par les lysosphingolipides, qui dérivent des sphingolipides correspondants par hydrolyse de l'acide gras couplé à la sphingosine. La protéine kinase C $(\mathrm{m} / \mathrm{s}$ $n^{\circ} 5$, vol. 1, p. 255) est très abondante dans le système nerveux. $\mathrm{Au}$ cours des sphingolipidoses, maladies dues à des déficits en enzymes des lysosomes qui dégradent les sphingolipides, les lysodérivés s'accumulent en même temps que les sphingolipides d'origine qui, par eux-mêmes, ne sont pas toxiques. Hannun et Bell proposent donc comme hypothèse générale l'inhibition de la protéine kinase $\mathrm{C}$ pour expliquer les troubles graves, souvent neurologiques, que provoquent ces maladies.

[Hannun YA, Bell RM. Science 1987 ; 235 : 670-4.] 